\title{
Simultaneous vibration and humidity measurement using a hybrid WDM/OCDMA sensor network
}

\begin{abstract}
In this paper a hybrid Wavelength Division Multiplexing (WDM) and Optical Code Division Multiple Access (OCDMA) sensor network is proposed to detect vibration and humidity simultaneously. Evaluation of the results for the proposed system is demonstrated using simulation setup. OCDMA is used for detection of vibration levels while WDM is for sensing humidity changes. Fiber Bragg gratings (FBGs) is used in both systems. In OCDMA FBGs were used as filter to form the OCDMA code and sensors to senses the vibration. In WDM FBGs were used as sensor alone to sense the humidity.
\end{abstract}

Keyword: Optical sensor network; Hybrid OCDMA/WDM; Vibration sensing; Humidity sensing 